\title{
INCORPORATION OF THE PORE SIZE VARIATION TO MODELING OF THE ELASTIC BEHAVIOR OF METALLIC OPEN-CELL FOAMS
}

\begin{abstract}
In the present paper we present the approach for modeling of the elastic behavior of open-cell metallic foams concerning non-uniform pore size distribution. This approach combines design of foam structures and numerical simulations of compression tests using finite element method (FEM). In the design stage, Laguerre-Voronoi tessellations (LVT) were performed on several sets of packed spheres with defined variation of radii, bringing about a set of foam structures with porosity ranging from 74 to $98 \%$ and different pore size variation quantified by the coefficient of pore volume variation, $\mathrm{CV}(\mathrm{V})$, from 0.5 to 2.1 . Each structure was numerically subjected to uni-axial compression test along three directions within the elastic region. Basing on the numerical response, the effective Young's modulus, $E_{\text {eff }}$, was calculated for each structure. It is shown that the $E_{\text {eff }}$ is not only dependent on the porosity but also on the pore size variation.
\end{abstract}

Keywords: open-cell metallic foams; Laguerre-Voronoi tessellations; pore size variation; Young's modulus

\section{Introduction}

Basically, the more uniform microstructure of materials, the higher mechanical properties. It has been broadly confirmed both for bulk and porous materials. In case of bulk materials, this uniformity concerns chemical composition and distribution of microstructural elements e.g. grain size, grain boundaries, precipitates etc. From the morphology point of view, porous materials contain two phases: solid matrix material and voids (pores), which in case of open-porosity are percolated. Thus in this case, structural uniformity is related mainly to the distribution of solid material and the pore size variation. Pores regarded as discontinuities of a solid matrix (bulk) are considered as a downside, from mechanical viewpoint, due to weakening of the material strength. As a consequence of depletion of solid in a cross-section, load-bearing capabilities of the structure is lowered. However, porous structures are inherently suitable for application as energy absorbers [1]. In the light of this fact, fraction of solid emerges as an essential factor determining mechanical behavior of porous materials [2,3]. Fraction of solid phase may be randomly distributed in the volume of porous material. In the literature one can find reports of characterization of foam structures or examination of its properties referred to the density distribution, by which the local non-uniformity is manifested [4].

Pore size variation in open-porous metals has measurable reflection in their properties. In the paper [5], it is shown that the pore size variation influences pressure drop of fluid flowing through a porous medium. In papers on the mechanics of cellular solids [3,6,7] authors presented numerical simulations of elastic properties of random metallic foams with non-uniform pore size. Pore size variation, however, is not strictly quantified there and no relationship between structural non-uniformity and elastic properties is derived basing on the computations. Analysis of the elastic behavior of open-cell structures was also performed in [2,8-12], but geometrically regular models were used, so thus non-uniformity was not incorporated to computations. In turn, the influence of pore shape regularity was analyzed in [13].

Complex structures of open-porous materials can be modeled by means of three methods:

- dimensional analysis based on a periodic alignment of regular and parameterized representative unit cells [2,11,14],

- Voronoi tessellation-based methods [14-17],

- reconstruction micro-computed tomography $(\mu \mathrm{CT})$ images $[3,18,19]$.

For the modeling of structures of sintered open-porous materials, a discrete approach incorporating the distribution of powder size may be successfully applied [20].

Although, as it is commonly established, mechanical properties of metallic open-cell materials strongly depend solely on the volume fraction of solid (relative density), we analyzed how pore size variation affects the elastic behavior of this materials. In the present study, a set of representative models of aluminum open-cell foams was designed with the porosity over the range from $74 \%$ to $98 \%$ and the coefficient pore volume of variation, $C V(V), 0.5$ and 2.1. Structures designed by means of LVT algo-

WARSAW UNIVERSITY OF TECHNOLOGY, FACULTY OF MATERIALS SCIENCE AND ENGINEERING, 141 WOŁOSKA STR., 02-507 WARSAW, POLAND

Corresponding author: karol.cwieka@inmat.pw.edu.pl 
rithm were then analyzed using FEM simulations of uni-axial compression along three orthogonal directions. Based on results of FEM analyses, the effective Young's modulus, $E_{\text {eff }}$, was calculated. Computational results were fitted to the Gibson-Ashby model to present the $E_{\text {eff }}$ as a function of the porosity and $C V(V)$.

\section{Materials and methods}

We designed several architectures of open-cell material using an algorithm based on Laguerre-Voronoi tessellations (LVT). The algorithm contains 3 stages: sphere packing, Laguerre-Voronoi tessellations and open-cell structure generation. The sphere packing stage was implemented in a software developed by the authors of this work and subsequent operations were coded using APDL language script implemented in the ANSYS software. The

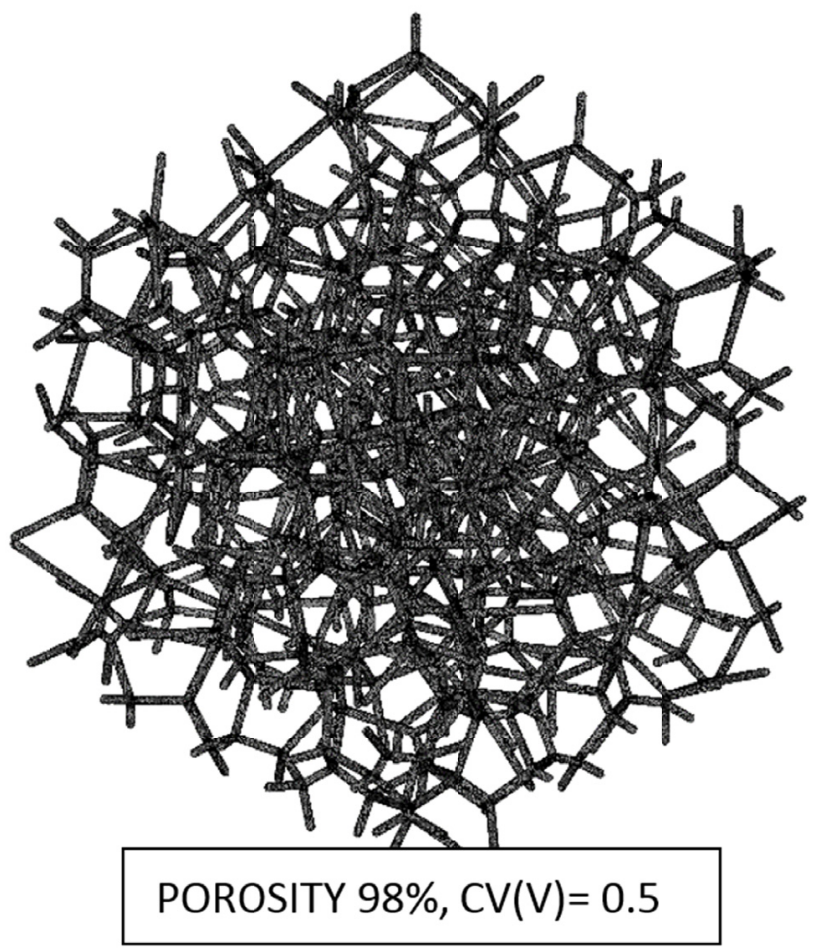

elastic properties of the foam material were assumed isotropic and defined by Young's modulus of $E=70 \mathrm{GPa}$ and Poisson's ratio $v=0.33$ for pure aluminum. Schematic explanation with detailed description of the procedure used hereby can be found in $[5,16,17]$.

By means of the above procedure we designed a set of 15 structures with different coefficient of pore volume variation, $C V(V)$. Values of $C V(V)$, determined as the ratio of the standard deviation to average pore volume, ranged between 0.5 to 2.1 . The highest uniformity of pore size distribution was obtained for the structure with $C V(V)=0.5$, while the highest diversity for $C V(V)=2.1$ one. In order to study the influence of porosity (relative density), the four values of strut diameter were analyzed bringing about structures with the lowest porosity about $74 \%$ and the highest about 98\%. Each structure was derived from 200 spheres (see example in (Fig. 1)).

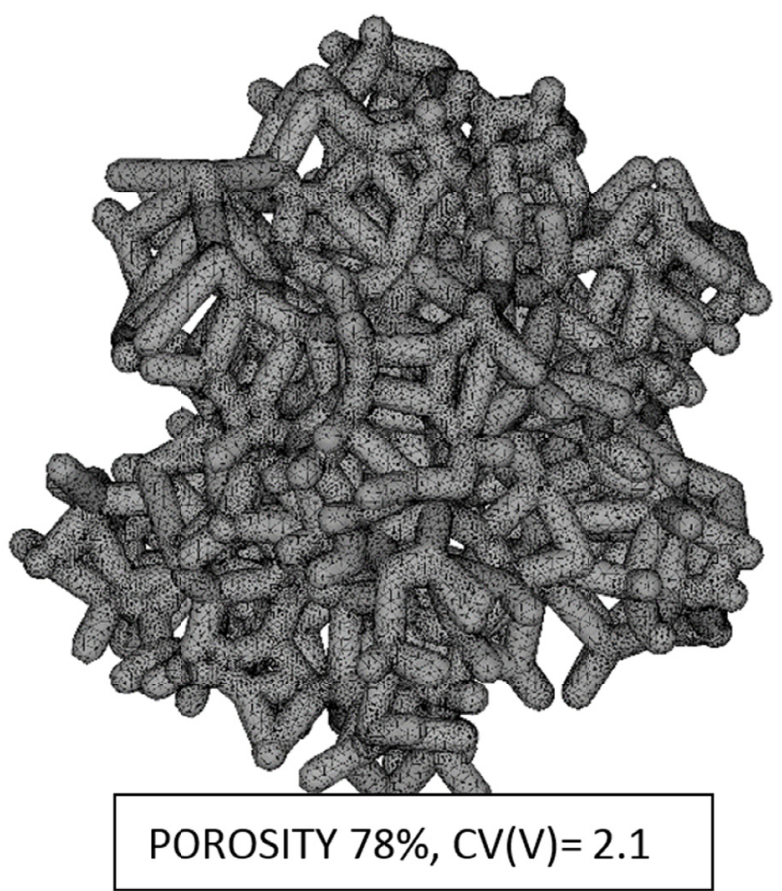

Fig. 1. Examples of designed structures of open-cell foams with different porosity and $C V(V)$

Elastic reactions of the designed structures were analyzed by means of FEM simulations of uni-axial compression along three orthogonal directions conducted using ANSYS software. Loading conditions corresponding to uni-axial compression test are presented in Figure 2.

Therefore, according to Hooke's law, effective Young's modulus $E_{\text {eff }}$ was calculated as:

$$
E_{\text {eff }}=\frac{\sigma_{\text {eng }}}{\varepsilon}=\frac{\frac{\Sigma F_{n}}{A}}{\frac{u_{n}}{a}}=\frac{\frac{\Sigma F_{n}}{a^{2}}}{\frac{u_{n}}{a}}=\frac{\Sigma F_{n}}{a u_{n}}
$$

where $\sigma_{\text {eng }}$ is engineering stress equal to sum of reaction forces $\Sigma F_{n}$ divided by an area of full cross-section $A, \varepsilon$ is deformation
- a quotient of displacement in normal direction un and initial edge length $a$.

The general scaling law for Young's modulus of open-cell materials has been proposed by Gibson and Ashby [21] as:

$$
\frac{E_{e f f}}{E_{S}}=C\left(\frac{\rho}{\rho_{S}}\right)^{n}
$$

where $E_{\text {eff }}$ is the effective Young's modulus of foam structure normalized with Young's modulus of bulk $E_{S}, \rho / \rho_{S}$ is relative density of foam and $C, n$ are constants dependent on the structure. Gibson-Ashby model was used here to verify correctness of computationally obtained relationship between $E_{\text {eff }}$ and relative density $\rho / \rho_{S}$. 


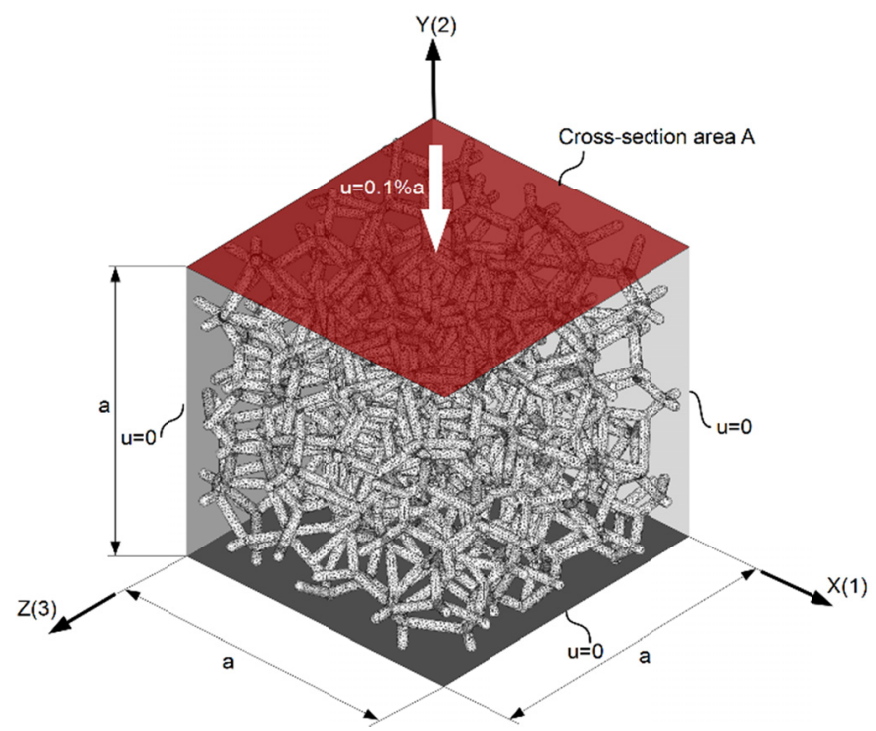

Fig. 2. Boundary conditions applied in FE simulations of uni-axial compression test

\section{Results and discussion}

The results were discussed in terms of the influence of pore size variation and the porosity on effective Young's modulus of open-cell metallic foams. Values of effective Young's modulus were calculated for each of three orthogonal directions of compression of designed aluminum open-cell foams and then averaged. The model derived by Gibson-Ashby (Eq. 2) was taken under consideration to represent a relationship between reduced Young's modulus and relative density of analyzed materials. To this end, computed values of $E_{\text {eff } A V G}$ were reduced by $E_{S}$ and plotted versus relative density $\rho / \rho_{S}$, in Figure 3 to show that Young's modulus is not only a function of relative density, but pore volume variation as well. The greater decrement of $E_{\text {eff }}$ is observable for lower porosity of approx. 74 to $84 \%$ (relative density 0.26 to 0.16 respectively).

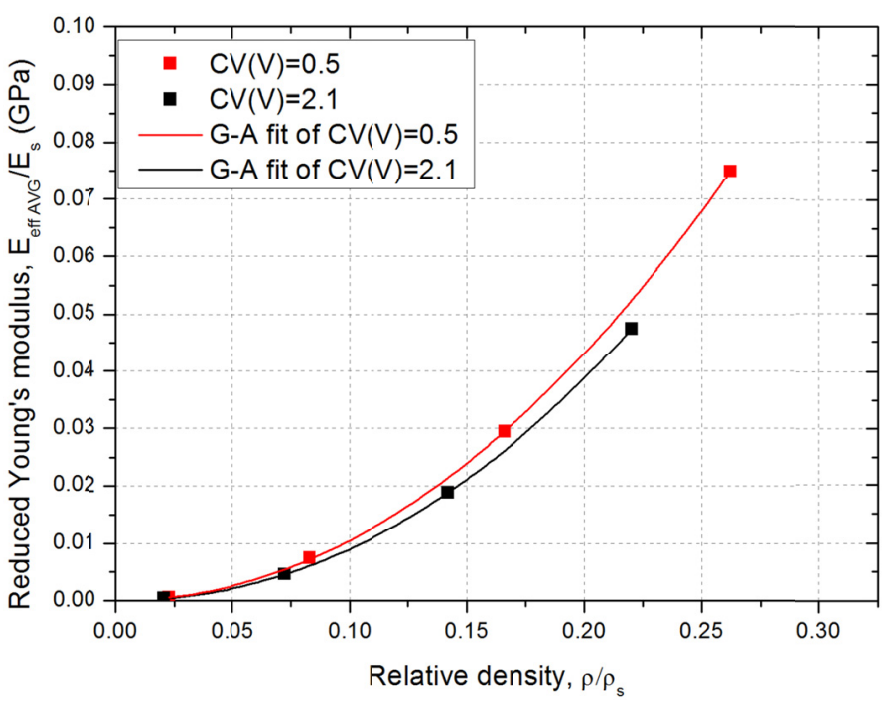

Fig. 3. Reduced values of average Young's modulus versus relative density of designed aluminum open-cell structures
The exponent $n$ is commonly assumed equal 2 for open-cell structures according to numerous experimental evaluations and it is considered a consequence of deformation mechanism. Computational data points fit excellent to G-A model $\left(R^{2}=0.99\right.$ for each series). A coefficient corresponding to scaling factor, $C$, is found out to be structure-related, and in the literature it is reported in the rage of 1.1-1.3. Analysis of data points computed using presented approach allows to infer, that the constant $C$ may be correlated with the coefficient of pore volume variation $C V(V)$. To completely prove that relationship, the extended set of structures should be designed and numerically analyzed in further studies.

\section{Conclusion}

The pore size variation was considered here as parameter affecting elastic properties of open-porous materials such as metallic open-cell foams. The coefficient of pore volume variation, $C V(V)$, was used to describe structural non-uniformity of architectures designed by means of Laguerre-Voronoi tessellationbased approach. Calculated values of effective Young's modulus, $E_{e f f}$, versus porosity perfectly fit the Gibson-Ashby model. We confirmed that the variation in size and geometrical arrangement of the cells is crucial for estimation of the mechanical properties of porous materials. According to performed computations, it is shown that $E_{\text {eff }}$ increases with decreasing porosity and decrease with increasing $C V(V)$ for the same porosity. Thus it was proven that the effective Young's modulus is a function of both porosity and structural inhomogeneity described with $C V(V)$. The presented approach may be successfully applied to broaden the investigation of the relationship of the structural non-uniformity and the elastic properties of metallic open-cell metals.

\section{REFERENCES}

[1] J. Banhart, Aluminium foams for lighter vehicles, Int. J. Veh. Des. 37, 114 (2005).

[2] R. Liu, A. Antoniou, A relationship between the geometrical structure of a nanoporous metal foam and its modulus, Acta Mater. 61, 7, 2390-2402 (2013).

[3] C. Veyhl, I.V. Belova, G.E. Murch, T. Fiedler, Finite element analysis of the mechanical properties of cellular aluminium based on micro-computed tomography, Mater. Sci. Eng. A 528, 13-14, 4550-4555 (2011).

[4] H. X. Zhu, J. R. Hobdell, A. H. Windle, Effects of cell irregularity on the elastic roperties of open-cell foams, Acta Mater. 48, 20, 4893-4900 (2000).

[5] J. Skibinski, K. Cwieka, T. Kowalkowski, B. Wysocki, T. Wejrzanowski, K.J. Kurzydlowski, The influence of pore size variation on the pressure drop in open-cell foams, Mater. Des. 87, 650-655 (2015).

[6] M. Geißendörfer, A. Liebscher, C. Proppe, C. Redenbach, D. Schwarzer, Stochastic multiscale modeling of metal foams, Probabilistic Eng. Mech. 37, 132-137 (2014). 
[7] X. Zhu, S. Ai, D. Fang, B. Liu, X. Lu, A novel modeling approach of aluminum foam based on MATLAB image processing, Comput. Mater. Sci. 82, 451-456 (2014).

[8] Y. An, C. Wen, P.D. Hodgson, C. Yang, Investigation of cell shape effect on the mechanical behaviour of open-cell metal foams, Comput. Mater. Sci. 55, 1-9 (2012).

[9] D. Edouard, M. Lacroix, C.P. Huu, F. Luck, Pressure drop modeling on SOLID foam: State-of-the art correlation, Chem. Eng. J. 144, 2, 299-311 (2008).

[10] A. Jung, S. Diebels, Modelling of Metal Foams by a Modified Elastic Law, Mech. Mater., 2016.

[11] G. Pia, F. Delogu, On the elastic deformation behavior of nanoporous metal foams, Scr. Mater. 69, 11-12, 781-784 (2013).

[12] J. Wieding, A. Wolf, R. Bader, Numerical optimization of open-porous bone scaffold structures to match the elastic properties of human cortical bone, J. Mech. Behav. Biomed. Mater. 37, 56-8 (2014).

[13] B. Drach, I. Tsukrov, A. Trofimov, Comparison of full field and single pore approaches to homogenization of linearly elastic materials with pores of regular and irregular shapes, Int. J. Solids Struct., (2016).

[14] A.P. Roberts, E.J. Garboczi, Elastic properties of model random three-dimensional open - cell solid 50, 2016, 33-55 (2002).
[15] I. Vecchio, C. Redenbach, K. Schladitz, A. M. Kraynik, Improved models of solid foams based on soap froth, Comput. Mater. Sci. 120, 60-69, Jul. (2016).

[16] T. Wejrzanowski, J. Skibinski, L. Madej, and K. J. Kurzydlowski, Modeling structures of cellular materials for application at various length-scales, Comput. Metods Mater. Sci. 13, 4, 493-500 (2013).

[17] T. Wejrzanowski, J. Skibinski, J. Szumbarski, K.J. Kurzydlowski, Structure of foams modeled by Laguerre-Voronoi tessellations, Comput. Mater. Sci. 67, 216-221 (2013).

[18] T. Fiedler, I. V. Belova, and G. E. Murch, " $\mu$-CT-based finite element analysis on imperfections in open-celled metal foam: Mechanical properties, Scr. Mater. 67, 5 455-458 (2012).

[19] T. Wejrzanowski, J. Skibinski, K. Cwieka, K.J. Kurzydlowski, Micro-computed tomography and finite element method study of open-cell porous materials, in MATEC Web of Conferences 30, (2015).

[20] T. Wejrzanowski, S.H. Ibrahim, K. Cwieka, J. Milewski, K.J. Kurzydlowski, Design of open-porous materials for high-temperature fuel cells, J. Power Technol. 96, 3 178-182 (2016).

[21] L.J. and M.F.A. Gibson, Cellular Solids. Cambridge University Press, 1997. 